Vassart pour isoler des séquences caractéristiques de ce type de récepteur : des amplifications par PCR à partir d'ADNc de muqueuse olfactive, en utilisant comme amorces des oligonucléotides correspondant à des régions très conservées des gènes codant pour les membres de cette grande famille de récepteurs $(\mathrm{m} / \mathrm{s}$ $n^{\circ} 2$, vol. 6, p. 166 et $n^{\circ} 6$, vol. 7, p. 634). Un très grand nombre de séquences pouvant correspondre aux molécules recherchées put ainsi être cloné ; 18 de ces clones furent complètement étudiés ; ils avaient tous le potentiel de coder pour des récepteurs à sept passages transmembranaires. Ces molécules peuvent être elles-mêmes subdivisées en sousfamilles dont les segments intramembranaires sont notoirement différents. Le site de fixation du ligand des récepteurs de cette super-famille, ici, par conséquent, de la molécule odorante, est situé entre les sept segments, dans le plan de la membrane. Ces sous-familles peuvent donc correspondre à la reconnaissance de types différents de molécules odorantes. Les modifications plus subtiles distinguant les différents membres d'une même sous-famille pourraient permettre la distinction entre des odeurs des dérivés d'une même molé- cule de base. On pourrait ainsi concevoir une sous-famille reconnaissant les molécules benzéniques, les différents membres distinguant le toluène du xylène, du phénol ou du benzène. L'utilisation d'un mélange des fragments amplifiés, correspondant à plusieurs sous-familles, comme sonde pour cribler une banque génomique permit d'apprécier à 200 copies par génome le nombre de clones reconnus. Ce chiffre est une estimation minimale de la diversité des gènes pouvant coder pour des récepteurs olfactifs. Certes, certains de ces clones génomiques pourraient contenir des pseudo-gènes et non des gènes, mais, en revanche, il est probable que la méthode utilisée a été incapable de reconnaître un grand nombre d'autres clones génomiques, codant pour des récepteurs d'autres sousfamilles. Les gènes de récepteurs olfactifs pourraient ainsi être au nombre de 200 à 1000 par génome. Cela suggère que les dix milliers d'odeurs reconnues par les mammiferes ne font pas appel à une combinatoire très complexe engendrée par l'utilisation d'un petit nombre de récepteurs formant un alphabet à quelques dizaines de lettres, mais s'apparente plutôt à des idéogrammes dans lesquels un symbole (c'est-à-dire un récepteur) - ou un très petit nombre de symboles - spécifie une odeur. Des études ultérieures diront si la perception par le cerveau des odeurs senties par la muqueuse olfactive se fait par un câblage simple en ligne, un neurone ou un très petit nombre de neurones centraux étant reliés à un ou à un très petit nombre de neurones périphériques sensoriels, ou si des processus plus complexes d'intégration existent au niveau cérébral. De tels processus seraient nécessaires si les neurones sensoriels exprimaient chacun une grande diversité de récepteurs, car il faudrait alors imaginer un système de tri des informations véhiculées par les axones, celles correspondant à des récepteurs donnés empruntant, par exemple, des branches particulières de l'arborisation terminale, les branches homologues de plusieurs axones convergeant vers un même groupe de neurones centraux. Les mécanismes possibles d'un tel tri restent très spéculatifs, et mieux vaut, à ce stade, attendre la suite des événements... avant tout des expériences d'hybridation in situ qui diront si les cellules sensorielles sont spécifiques d'un récepteur donné ou en expriment un grand nombre.

A. $\mathbf{K}$.

\title{
口ᄆ BRÈVES
}

Petites protéines G, mitoses et transit intracellulaire. Entre autres nombreuses modifications associées à la division cellulaire, l'inhibition des phénomènes d'endocytose et d'exocytose semble liée à l'activité de la protéine kinase $\mathrm{p} 34^{\mathrm{cdc} 2}$ [1]. On sait depuis environ deux ans que les différentes étapes du transit intracellulaire des protéines, qu'il s'agisse de processus sécrétoires ou d'endocytose, sont contrôlées par des G-protéines. Chez la levure, deux étapes distinctes du processus sécrétoire sont contrôlées par des petites protéines $G$ appartenant à la famille Ras, Sec4p et Ypt1p. Chez les mammifères, existe un très grand nombre de petites protéines $G$ de cette famille, parmi lesquelles les lecteurs de $\mathrm{m} / \mathrm{s}$ connaissent déjà la protéine $m / s n^{\circ} 6$, vol. 7 , juin-juillet 91
Rap1A/Krev1 qui est localisée au niveau du complexe de Golgi $(\mathrm{m} / \mathrm{s}$ $n^{\circ}$ 5, vol. 7, p. 518). Diverses protéines $\mathrm{Rab}$ sont également localisées dans des compartiments distincts des voies endocytiques et exocytiques. Des chercheurs du centre Cnrs de Gif-sur-Yvette, de l'Institut Pasteur de Paris et du CHU Lariboisière Saint-Louis ont voulu tester l'hypothèse selon laquelle la modification de protéines Rab au cours du cycle cellulaire était responsable de l'inhibition des phénomènes de transit [2]. De fait, deux protéines Rab (Rab1Ap et Rab4p) sont spécifiquement phosphorylées par la protéine kinase p34 cdc2 dans sa forme activée, compétente pour déclencher la mitose. D'autres protéines de cette même famille Rab ne sont pas phosphory- lables. La phosphorylation des protéines Rab1Ap et Rab4p modifie également leur répartition intracellulaire, la phosphorylation tendant à mener l'une vers la membrane et l'autre vers le cytosol. En conclusion, le chef d'orchestre de la division cellulaire qu'est la kinase p34 cdc2 inhibe les transports intracellulaires au cours de la mitose, cet effet étant probablement relayé, au moins en partie, par une phosphorylation de petites protéines $G$ qui jouent un rôle essentiel dans le contrôle de ces phénomènes de transit intracellulaire.

[1. Cavadore JC. médecine/sciences $1990 ; 6$ : 895-900.]

[2. Bailly $\mathrm{E}$, et al. nature $1991 ; 350$ 715-8.]

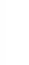

